\title{
Demonstrating the feasibility of collecting secondary, de-identified data on Australian patients receiving treatment as part of a Medicine Access Program
}

Lok Sheau Wen ${ }^{1,3}$, De Boer Richard ${ }^{3}$, Cordwell Cassandra ${ }^{4}$, Marx Gavin ${ }^{5}$, Fox Peter ${ }^{6}$, Hasovits Csilla ${ }^{5}$, Rutovitz Joseph ${ }^{7}$ Harold Michael $^{1}$, Tran Ben ${ }^{1,3}$, Wong Hui-Li ${ }^{1,3}$ and Gibbs Peter ${ }^{1,2}$.

1. Walter and Eliza Hall Institute, Parkville, Melbourne, VIC

2. University of Melbourne, Parkville, Melbourne, VIC

3. Peter MacCallum Cancer Centre, Parkville, Melbourne, VIC

4. Roche Products Pty Limited, Sydney, NSW,

5. The University of Sydney and Sydney Adventist Hospital, Wahroonga, NSW

6. Orange Health Service, Orange, NSW

7. Northern Haematology \& Oncology Group, Wahroonga, NSW

\section{Corresponding Author}

Sheau Wen Lok

Systems Biology and Personalised Medicine Division, The Walter and Eliza Hall Institute of Medical Research, Level 8 Royal Melbourne Hospital, Grattan St, Parkville, VIC 3050 Australia Sheauwen.lok@petermac.org

03-93452893

\section{Word count}

Abstract: 250

Main text: 2288

\section{ABSTRACT}

Background: In Australia, data generated from the carefully selected, treated and monitored patients enrolled in clinical trials largely inform routine care and funding approvals. Medicine Access

This is the author manuscript accepted for publication and has undergone full peer review but has not been through the copyediting, typesetting, pagination and proofreading process, which may lead to differences between this version and the Version of Record. Please cite this article as doi: 10.1111/imj.14265

This article is protected by copyright. All rights reserved. 
Programs (MAPs) enable drug access and whilst potentially a rich source of data, historically have not collected data beyond a participant list.

Aims: To explore the feasibility of using MAPs to identify patient populations for inclusion in noninterventional studies.

Methods: Clinicians affiliated with the Walter and Eliza Hall Institute (WEHI) engaged with Roche to implement PeRSIA, a secondary data use non-interventional study of patients receiving neoadjuvant pertuzumab for non-metastatic HER2+ breast cancer. The study utilised a pre-existing Rochesponsored MAP to identify clinicians as data contributors. Data security, ownership and reporting issues were addressed utilising the BioGrid platform and standards developed for existing WEHI registries. Disease experts developed project-specific Case Report Forms documenting treatment, surgical and cancer-specific outcomes, and adverse events.

Results: To date, 12 of 16 (75\%) clinicians approached to participate in PeRSIA are contributing deidentified data. From February through September 2018, data on 41 patients from 7 centres were collected. Median patient age is 56 years (range 36-81), 36 (88\%) had Stage 2 to 3 disease, and 27 (66\%) were node positive. The median number of cycles of neoadjuvant pertuzumab planned was 4 .

Conclusion: This initial report is, to our knowledge, the first description of a secondary data use noninterventional study collecting comprehensive data on patients enrolled, independently, in a MAP. This effort continues and opportunities with other industry partners are being pursued.

Key words - Breast Cancer; Non-interventional study; access program; Real world data

\section{Introduction}

Rapid progress in cancer research has seen the emergence of many new therapeutic options. Equitable and sustainable access to these underpins Australia's national medicine policies, with the aim of subsidising access to medicines that are deemed cost effective. In the interval between initial publication of trial results and the approvals that then make these new agents broadly available, Medicine Access Programs (MAPs) enable patient access to new medicines.

The addition of pertuzumab to trastuzumab (plus chemotherapy) in the neoadjuvant setting for patients with early stage and locally advanced HER2+ breast cancer results in higher rates of pathological complete response. ${ }^{1}$ It is not yet known if this translates to improved survival 
outcomes. Pertuzumab was approved in Australia (May 2016) by the Therapeutic Goods Administration (TGA), to be given in combination with trastuzumab and chemotherapy for the neoadjuvant treatment of locally advanced and inflammatory HER2+ breast cancer. This patient group can now access pertuzumab through a Roche MAP where pertuzumab is provided to eligible patients in a cost share program.

Despite the potentially rich data that could be generated from MAPs, including safety and efficacy data in a more 'real world' population, to date this opportunity has not been pursued. This is despite strong advocacy for this over an extended period, both locally ${ }^{2,3}$ and internationally $y^{4-6}$. The failure to initiate such a data collection effort we would suggest reflects the many associated challenges, as summarised in Table 1.

In recent years, clinician researchers at WEHI and BioGrid Australia ${ }^{7}$ have successfully implemented multi-site, national and international clinical registries for many tumour types. These go well beyond standard clinical registries that focus on audit and quality data at the time of initial diagnosis, with the intent of the WEHI-sponsored registries being to capture comprehensive patient and tumour detail, multidisciplinary treatment delivered and the outcomes across all lines of therapy. The value of these efforts has been demonstrated in the research output, ranging from early diagnosis and screening ${ }^{8}$ through to management across all lines of therapy for advanced disease ${ }^{9}$. More recently a particular focus has been the utilisation of this clinical data in translational research projects, including multiple blood ${ }^{10}$ and tumour biomarker studies. The latter have included both academic collaborations, such as Jorrisen et al ${ }^{11}$ and commercial collaborations, such as Ghosh et al. ${ }^{12}$ Ultimately this effort at multi-centre data collection and analysis has over time identified and addressed the many challenges common to data collection as part of an MAP.

Here we describe the implementation and initial experience of PeRSIA (Pertuzumab study for HER2 positive non-metastatic breast cancer in the neoadjuvant setting in Australia), a secondary data use non-interventional study of this patient population, built on the BioGrid platform. We describe the population of patients enrolled in PeRSIA so far and discuss some of the many potential uses of this data, including in support of funding applications and further research efforts focused on real world outcomes.

\section{Methods}

PeRSIA (ML39622) is a secondary data use non-interventional study of Australian patients initiating or considering pertuzumab treatment in the neoadjuvant setting for non-metastatic HER2+ breast

This article is protected by copyright. All rights reserved. 
cancer. The primary objective of the study is to capture real world data on the safety and effectiveness of pertuzumab in the neoadjuvant setting. Clinicians who prescribe TGA approved neoadjuvant pertuzumab through a Roche-supported cost share access program (http://www.rocheaccessprograms.com.au) are invited by the Roche study team to participate in the study and contribute de-identified patient data. On agreement to participate, the clinicians' contact details are then passed by the Roche study team to the PeRSIA management team at WEHI.

The project was initiated following an agreement between Roche and WEHI, with support from expert breast cancer clinicians, a protocol for the PeRSIA study was developed. Policies related to data ownership for initiating a research project and for co- authorship on any research output are consistent with the established standards for WEHI clinical registries. As the sponsor of the study, Roche has the right to publish the methods, results and conclusions, with co-authorship as per the ICMJE criteria. Ethical approval was initially obtained from the human research and ethics committee at Melbourne Health who advised that patient informed consent was not required due to the de-identified nature of the data collected, in accordance with the Guidelines Under Section 95 of the Australian Privacy Act 1988 (March 2000). As the study rolled out to other sites, an opt-out consent approach was required and implemented at several sites according to guidance from the local ethics committee.

The case report form (CRF) for PeRSIA was developed by a team of experienced clinicians with joint appointments at WEHI and various public and private hospitals in Victoria, in conjunction with Roche. An electronic database was then created using the REDCap (Research Electronic Data Capture) application ${ }^{13}$, a mature, secure, open source tool hosted in the WEHI data centre, which is protected through a site-specific unique username and password system.

Patient and tumour characteristics, treatment details (including surgery, chemotherapy, HER2 targeted therapy and radiation therapy), safety data, pathological response and early recurrence data are to be captured at various time points over an 18-month period (Figure 1). These four specific time points correspond to 1 ) prior to the patient commencing neoadjuvant therapy, 2) after completion of neoadjuvant therapy and before surgery, 3 ) one to two months post-surgery and 4) eleven to fourteen months post-surgery. Data will be retrieved from medical notes by the treating physician and entered on to the CRF with a unique patient identifier assigned to each patient. The average completion time for each CRF is 15 minutes. De-identified data is transferred electronically to WEHI, manually entered into REDCap by WEHI staff and aggregated for analysis purposes. Data quality is regularly monitored by WEHI study personnel and feedback provided to the participating centres.

The co-primary endpoints of the study are the incidence of adverse events related to pertuzumab, breast pathological complete response rate $(p C R)$ and total $p C R$. Secondary objectives include rates 
of mastectomy, relapse-free survival and overall survival 12 months post-surgery, as well as any discrepancy between planned and actual therapy administered, including chemotherapy and HER2 targeted agents. The initial recruitment target is 80 patients, based on an estimate of the number of Australian patients per year who are anticipated to fit the indication and able to fund the cost of treatment. From a safety perspective, the probability of observing at least one event of left ventricular systolic dysfunction (LVSD) with 80 patients exceeds $99 \%$ based on the TRYPHAENA study. ${ }^{14}$

An additional 25 patients receiving neoadjuvant therapy without pertuzumab will also be recruited as a separate cohort. Data from these patients will enable an evaluation of patient demographic and disease characteristics for this cohort, as well as an understanding of the reasons why some patients in the real world setting do not go on to receive neoadjuvant pertuzumab. Data analysis will be performed using $\mathrm{SAS}^{\circledR}$ querying, analytical and reporting tools. Data from participating centres will be combined for analysis and reporting.

\section{Results}

As of September 2018, 12 of the 16 clinicians (75\%) approached by the WEHI study management team have proceeded with study participation. Discussion is continuing with three of the other clinicians. One site has formally declined to participate due to the small number of patients that were receiving treatment across a number of different health services, necessitating a significant amount of administrative and governance resources. Data has now been received for 41 patients from seven sites in the 8 months that the study has been open. Patient recruitment to the PeRSIA registry is illustrated in Figure 2.

The median age of patients entered in the PeRSIA registry is 56 years (range $35-81$ years). Most cancers were stage $2(59 \%)$ and $3(29 \%)$, and node positive (66\%) at the time of diagnosis. Estrogen receptor status was positive in 21 patients (51\%). The median number of cycles of neoadjuvant pertuzumab and trastuzumab planned were 4 and 5 respectively. Eighteen patients (44\%) were planned to receive combination chemotherapy with an anthracycline and taxane (e.g. AC/ECPaclitaxel), $22(54 \%)$ to receive single agent paclitaxel and $1(2 \%)$ to receive a combination of carboplatin and docetaxel. Data on the actual number of cycles of HER2 targeted therapy and type of chemotherapy received is pending.

\section{Discussion}

This article is protected by copyright. All rights reserved. 
In Australia, decisions to fund a new medicine, or an approved medicine in a new indication, are based on a rigorous 'value for money' assessment. This follows an initial evaluation of the available efficacy and safety data, the great bulk of which is generated from clinical trials. As funding approvals may take several years, MAPs provide an interim mechanism for patients to access and benefit from the new treatment option. MAPs may enroll many hundreds of patients and have the potential to generate much valuable and timely outcome data, including important insights into the real world impact of a new medicine. Historically however, very limited data has been collected in MAPs.

To our knowledge there is only one previous report of data collected as part of an Australian MAP, where cetuximab was provided to patients with metastatic colorectal cancer on a cost share basis. Although limited by a selected dataset, this analysis by Tie et al ${ }^{2}$ enabled exploration of the impact of patient socioeconomic status, age and gender on MAP participation. In keeping with the findings from Tie et al, preliminary data from the first 41 patients enrolled in the PeRSIA study indicates that this is a relatively young population (median age of 57 years). The further pathology and planned treatment data presented illustrates the depth of data that is being captured, including tumor characteristics and planned treatment. An initial abstract describing PeRSIA and some preliminary data on patient enrollment was presented at a local breast cancer conference ${ }^{15}$. A complete study report will be generated at the completion of the study and several publications are planned to report the outcomes of the study objectives.

This Roche-sponsored activity is built upon a much larger effort to collect comprehensive treatment and outcome data in routine care, led by clinicians with joint clinical and WEHI appointments, now extending to 10 registries across many cancer types. Built on the BioGrid Australia platform these registries extend to more than 40 sites nationally and internationally, including sites through the Asia Pacific. This activity is summarized in Figure 3. The clinical registries team at WEHI, consisting of clinicians, program managers, computer programmers, data officers, statisticians and ethics coordinators, now has extensive experience in developing datasets, creating databases with intuitive user interfaces and navigating ethics and governance requirements at multiple sites. Standards for data access and ownership, as well as authorship policies for any generated research output, are agreed upon by clinicians before participating in data collection. These efforts have now systematically addressed issues relevant to collecting data as part of a MAP, which now combined with an industry sponsored non-interventional study through a third party, makes projects such as PeRSIA feasible for the first time.

Part of the success of PeRSIA can perhaps be attributed to clinicians experiencing previous successful data-related collaborations with Roche, including a metastatic colorectal cancer registry, $\operatorname{TRACC}^{16}$, and a metastatic HER2+ breast cancer registry, TABITHA. ${ }^{17}$ Regardless, to fully engage clinicians in data collection for a project like PeRSIA, the incentives must compensate for the work involved. The modest per patient payments do provide some compensation for the time and effort required to achieve ethics approval and to contribute initial and follow-up data. Anecdotally, further incentives 
for clinician engagement in PeRSIA include contributing to research efforts related to a drug used in a relatively new and exciting context, and providing the clinical community with additional efficacy and safety data in a real world setting. PeRSIA will also generate multiple publications and authorship opportunities for contributing clinicians. While not a part of PeRSIA, it is envisaged that future efforts at MAP-associated data collection could include ethical approval for tissue-based research on archival samples, as is the case for many existing WEHI-supported registries, enabling future translational research opportunities.

From a pharmaceutical sponsor's perspective, there are several benefits of real world data generation including optimising medical practice, accelerating and sustaining patient access and innovating development. Real world data allows for an effective response to regulators' and payers' demands by providing proof of real world effectiveness. It can also reduce the cost of data generation and help inform clinical development programs, such as helping to select specific patient populations for future studies, especially those that are typically underrepresented in clinical trials. Along with adverse event data, early outcome data can be explored. This includes surgical outcomes for the PeRSIA population, such as pathologic complete response rates, a surrogate for risk for recurrence. Where supported, the collection of longer-term outcome data, including maturing progression-free and overall survival data, would add significant value when looking to fully understand treatment impact.

While we are not reporting any outcome data at this point, it is appropriate to consider any potential risks associated with data collection efforts such as PeRSIA. A formal protocol should be established and study objectives defined for each project, with all endpoints to be fully reported, including any unfavourable outcomes. Where outcome data is particularly favourable, this must be carefully interpreted, given the modest sample size and lack of a control population. Although the expectation is that outcomes will be inferior in a real world setting compared to those achieved in clinical trials, in any instance where unexpectedly good results are achieved these should not be used in place of clinical trial data to promote use of the drug.

As with most observational cohort studies, data collection alongside MAPs has multiple limitations and these should be clearly stated at the time of data reporting and publication. These include uncertain data quality as this is dependent on the accuracy and completeness of medical records, and is unlikely to meet the high standards set by randomised controlled trials. Rather MAP related data should be used to provide reassurance that treatment benefit and adverse effects in a real world population are in line with those reported in clinical trials. MAP data may also potentially fill in some of the knowledge gaps, for example where randomised controlled trials have not addressed outcomes in a specific patient subset of interest.

\section{Conclusion}

This article is protected by copyright. All rights reserved. 
To our knowledge we have described the first joint effort between clinicians and a pharmaceutical industry sponsor to enable the collection of de-identified, comprehensive data from patients receiving treatment via a MAP. We have explored the potential value of such data, including how this could inform consideration related to approvals of new drugs or a new funding indication. There is also substantial further research potential, including possible translational studies. The experience with PeRSIA is relevant, well beyond this medicine, beyond this tumor type and beyond medical oncology, as new and inevitably expensive medicines are being developed across a broad range of diseases. In each case we would assume that additional data on the effectiveness and the safety of these agents in a real world population would be of great interest to funders, to pharmaceutical companies and to the clinical community. We are actively exploring other opportunities and would encourage the broader medical community to do the same based on our initial positive experience.

\section{Acknowledgements}

The PeRSIA study is sponsored by Roche Products Pty Limited. We would also like to thank Siavash Foroughi for developing the REDCap electronic database for the PeRSIA study.

This article is protected by copyright. All rights reserved. 


\section{References}

1. Gianni L, Pienkowski T, Im YH, Roman L, Tseng LM, Liu MC, et al. Efficacy and safety of neoadjuvant pertuzumab and trastuzumab in women with locally advanced, inflammatory, or early HER2-positive breast cancer (NeoSphere): a randomised multicentre, open-label, phase 2 trial. Lancet Onc. 2012; 13: 25-3.

2. Tie J, Gibbs P. Treatment with unfunded drugs in oncology: the impact of access programmes and clinical trials. Intern Med J. 2013; 43: 23-31

3. Grover P, Babar ZU, Oehmen R, Vitry A. Medicines access programs to cancer medicines in Australia and New Zealand: An exploratory study. Health Policy. 2018; 122: 243-249

4. Chisholm MA, DiPiro JT. Pharmaceutical manufacturer assistance programs. Arch Intern Med 2002; 162: 780-4.

5. Felder TM, Lal LS, Bennett CL, Hung F, Franzini L. Cancer patients' use of pharmaceutical patient assistance programs in the outpatient pharmacy at a large tertiary cancer center. Community Oncol. 2011; 8: 279-286.

6. Kletas V, de Lemos M. Development of a systematic approach to pharmaceutical industry's patient assistance programs on accessing unfunded cancer drugs. J Oncol Pharm Pract. 2018;

24:18-21

7. Merriel RB, Gibbs $P, O^{\prime}$ Brien TJ, Hibbert M. BioGrid Australia facilitates collaborative medical and bioinformatics research across hospitals and medical research institutes by linking data from diverse disease and data types. Hum Mutat. 2011; 32: 517-25

8. Ananda S, McLaughlin S, Chen F, Hayes I, Hunter A, Skinner I, et al. Initial impact of the Australian National Bowel Cancer Screening Program. MJA 2009; 191: 378-381.

9. Lim L, Gibbs P, Yip D, Shapiro J, Dowling R, Smith D, et al. A prospective evaluation of treatment with Selective Internal Radiation Therapy (SIR-spheres) in patients with unresectable liver metastases from colorectal cancer previously treated with 5-FU based chemotherapy. Biomed Central. 2005; 5: 132-137.

10. Cohen J, Li L, Wang Y, Thoburn C, Afsari B, Danilova L, et al. Detection and localization of surgically resectable cancers with a multi-analyte blood test. Science 2018; 359: 926 - 930.

This article is protected by copyright. All rights reserved. 
11. Jorissen R, Gibbs P, Christie M, Prakash S, Lipton L, Desai J, et al. Metastasis-associated gene expression changes predict recurrence in patients with stage II and stage III colorectal cancer. Clin Cancer Res 2009; 15: 7642-7651.

12. Ghosh P, Tie J, Muranyi A, Singh S, Brunhoeber P, Leith K, et al. Girdin (GIV) Expression as a Prognostic Marker of Recurrence in Mismatch Repair Proficient Stage II Colon Cancer. Clin Cancer Res 2016; 22: 3488-98.

13. Harris P, Taylor R, Thielke R, Payne J, Gonzalez N, Conde J. Research electronic data capture (REDCap) - A metadata-driven methodology and workflow process for providing translational research informatics support. J Biomed Inform. 2009; 42: 377-81.

14. Schneeweiss A, Chia S, Hickish T, Harvey V, Eniu A, Waldron-Lynch M, et al. Long-term efficacy analysis of the randomised, phase II TRYPHAENA cardiac safety study: Evaluating pertuzumab and trastuzumab plus standard neoadjuvant anthracycline-containing and anthracycline-free chemotherapy regimens in patients with HER2-positive early breast cancer. European Journal Cancer. 2018; 89: 27-35.

15. Deboer R, Lok S, Harold M, Gibbs P, Cordwell C. Design of PeRSIA: Pertuzumab study for HER2positive non-metastatic breast cancer in the neoadjuvant setting in Australia. Melbourne International Joint Breast Cancer Congress, Melbourne, October 11-13, 2018

16. Field K, Wong HL, Shapiro J, Kosmider S, Tie J, Bae S, et al. Developing a national database for metastatic colorectal cancer management: perspectives and challenges. Intern Med J. 2013; 43:1224-31

17. Lok SW, Pellegrini L, Greenberg S, Oakman C, Green M, Foo S, et al. TABITHA - A prospective multi-site registry capturing treatment and outcome data in the metastatic HER2-positive breast cancer patient. APJCO 2016; 12: 60.

This article is protected by copyright. All rights reserved. 
TABLES

- Ethics approval and patient consent

- Identification of participating clinicians and patients

- Clinician engagement, reward and acknowledgement

- Defining datasets, data dictionaries and developing databases

- Data privacy, security, ownership

- Data entry and reporting of adverse events

- Data quality and analysis

Table 1: Some of the many challenges to be addressed to enable MAP data collection

This article is protected by copyright. All rights reserved. 


\section{FIGURE LEGENDS}

Figure 1. PeRSIA schema and data capture timelines. Data to be collected at four time points on paper case report forms (CRFs) by the treating clinician.

Figure 2. Participating sites and recruitment over time

Figure 3. Existing WEHI multi-centre cancer registries.

This article is protected by copyright. All rights reserved. 


\section{ABSTRACT}

Background: In Australia, data generated from the carefully selected, treated and monitored patients enrolled in clinical trials largely inform routine care and funding approvals. Medicine Access Programs (MAPs) enable drug access and whilst potentially a rich source of data, historically have not collected data beyond a participant list.

Aims: To explore the feasibility of using MAPs to identify patient populations for inclusion in noninterventional studies.

Methods: Clinicians affiliated with the Walter and Eliza Hall Institute (WEHI) engaged with Roche to implement PeRSIA, a secondary data use non-interventional study of patients receiving neoadjuvant pertuzumab for non-metastatic HER2+ breast cancer. The study utilised a pre-existing Rochesponsored MAP to identify clinicians as data contributors. Data security, ownership and reporting issues were addressed utilising the BioGrid platform and standards developed for existing WEHI registries. Disease experts developed project-specific Case Report Forms documenting treatment, surgical and cancer-specific outcomes, and adverse events.

Results: To date, 12 of 16 (75\%) clinicians approached to participate in PeRSIA are contributing deidentified data. From February through September 2018, data on 41 patients from 7 centres were collected. Median patient age is 56 years (range 36-81), 36 (88\%) had Stage 2 to 3 disease, and 27 (66\%) were node positive. The median number of cycles of neoadjuvant pertuzumab planned was 4.

Conclusion: This initial report is, to our knowledge, the first description of a secondary data use noninterventional study collecting comprehensive data on patients enrolled, independently, in a MAP. This effort continues and opportunities with other industry partners are being pursued.

Key words - Breast Cancer; Non-interventional study; access program; Real world data 
FIGURE 1

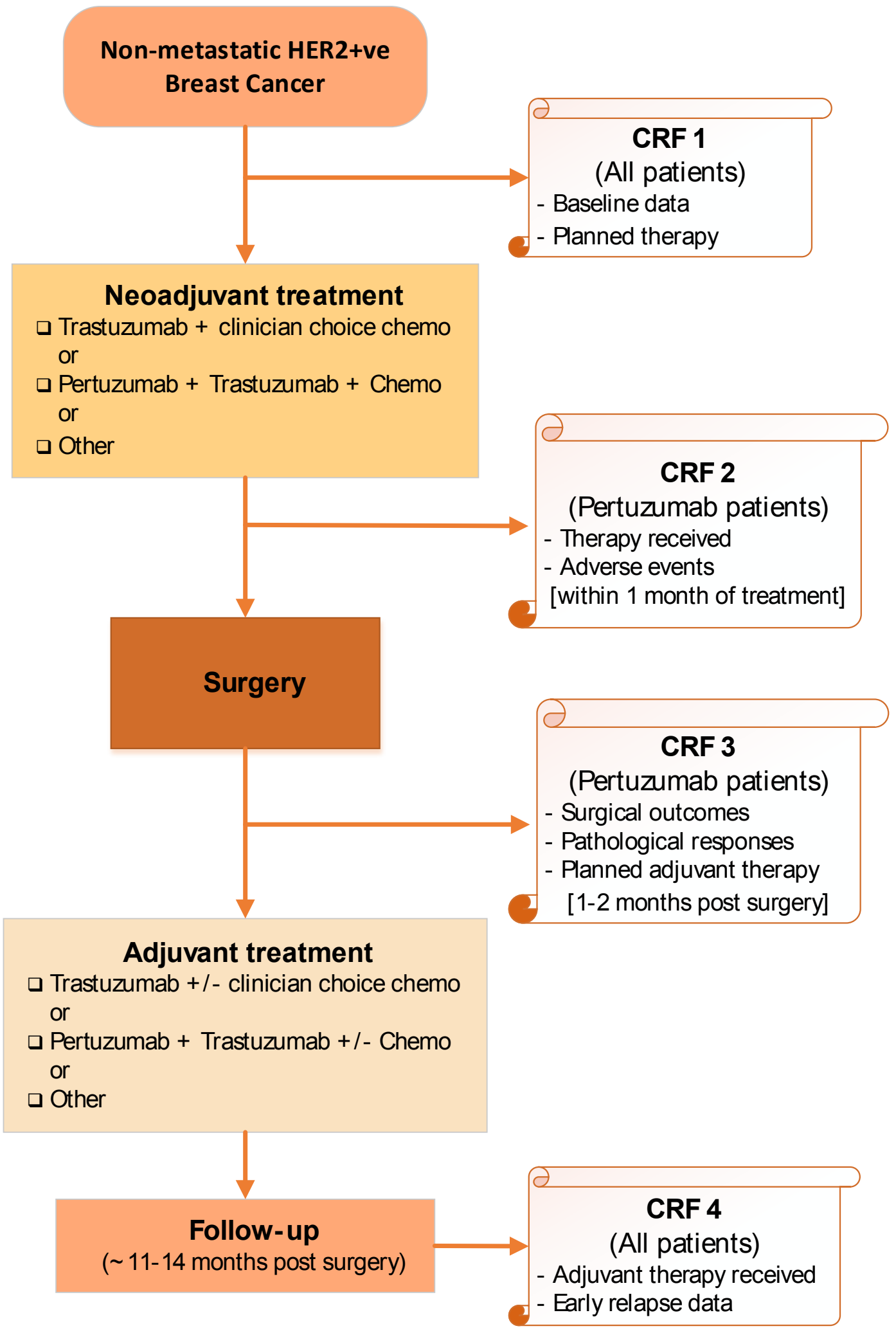

This article is protected by copyright. All rights reserved. 
FIGURE 2

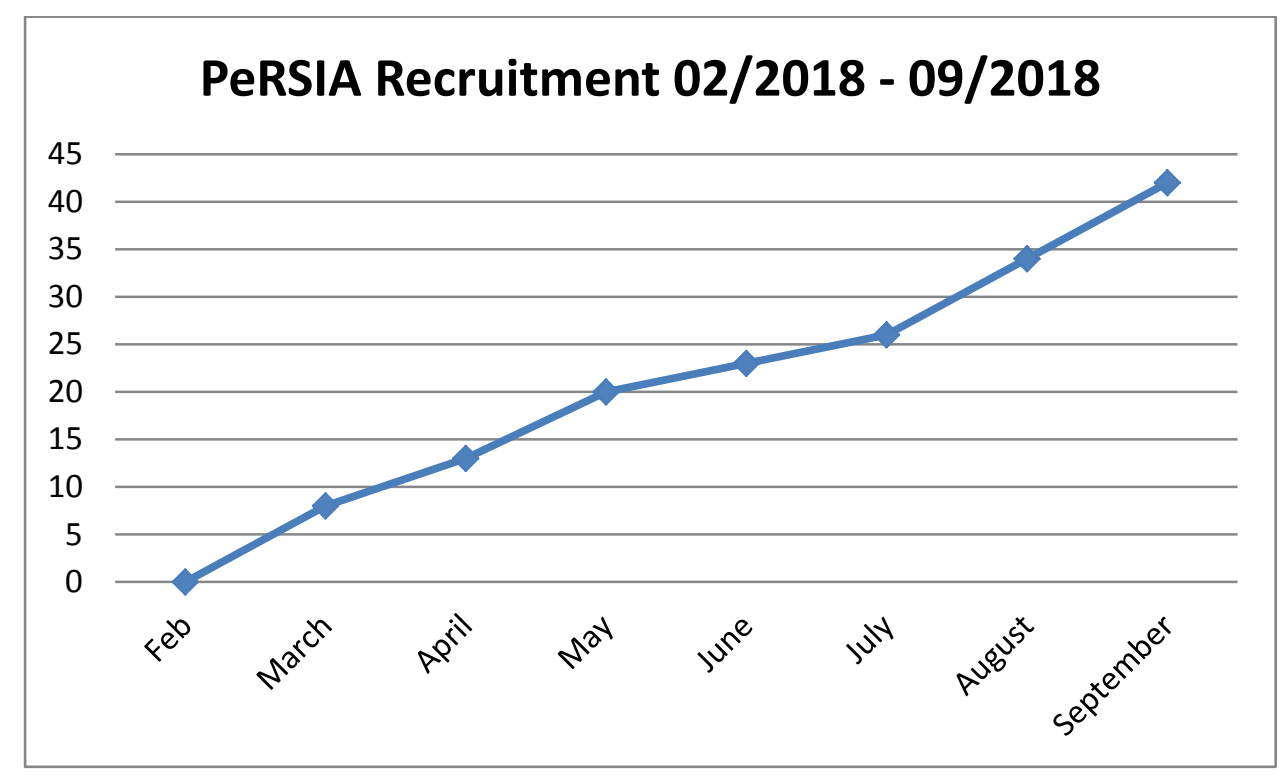

This article is protected by copyright. All rights reserved. 
FIGURE 3

Walter+Eliza Hall Discovenies ton nuvasiry

ACTIVE
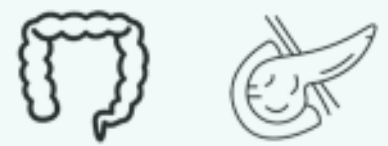

TRACC MCRC

PURPLE

Pancreatic cancer

IN DEVELOPMENT

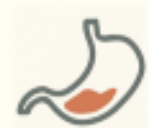

GATOR

Gastro-

esophageal cancer

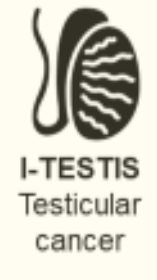

WEHI-BioGrid Clinical Registries
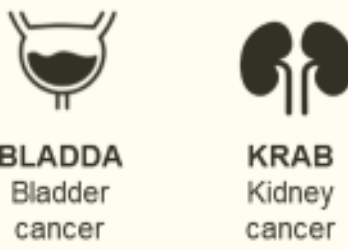

OTHER
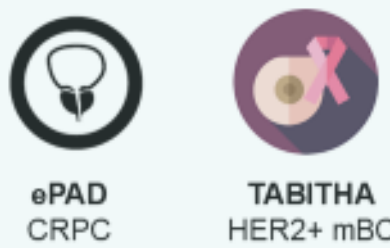

TABITHA

$\mathrm{HER} 2+\mathrm{mBC}$

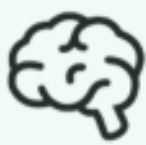

BRAIN

Gliomas

Pertuzumab in HER2+ nonmetastatic BC

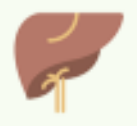

RESIN

Y-90 resin microspheres

This article is protected by copyright. All rights reserved. 


\section{Demonstrating the feasibility of collecting secondary, de-identified data on Australian patients receiving treatment as part of a Medicine Access Program}

Lok Sheau Wen ${ }^{1,3}$, De Boer Richard ${ }^{3}$, Cordwell Cassandra ${ }^{4}$, Marx Gavin ${ }^{5}$, Fox Peter ${ }^{6}$, Hasovits Csilla ${ }^{5}$, Rutovitz Joseph ${ }^{7}$ Harold Michael $^{1}$, Tran Ben ${ }^{1,3}$, Wong Hui-Li ${ }^{1,3}$ and Gibbs Peter ${ }^{1,2}$.

1. Walter and Eliza Hall Institute, Parkville, Melbourne, VIC

2. University of Melbourne, Parkville, Melbourne, VIC

3. Peter MacCallum Cancer Centre, Parkville, Melbourne, VIC

4. Roche Products Pty Limited, Sydney, NSW,

5. The University of Sydney and Sydney Adventist Hospital, Wahroonga, NSW

6. Orange Health Service, Orange, NSW

7. Northern Haematology \& Oncology Group, Wahroonga, NSW

\section{Corresponding Author}

Sheau Wen Lok

Systems Biology and Personalised Medicine Division, The Walter and Eliza Hall Institute of Medical Research, Level 8 Royal Melbourne Hospital, Grattan St, Parkville, VIC 3050 Australia

Sheauwen.lok@petermac.org

03-93452893

\section{Word count}

Abstract: 250

Main text: 2288

This article is protected by copyright. All rights reserved. 


\section{University Library}

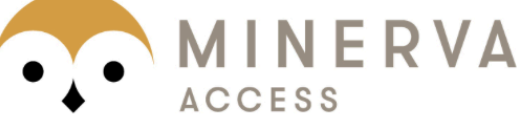

A gateway to Melbourne's research publications

Minerva Access is the Institutional Repository of The University of Melbourne

Author/s:

Lok, SW;De Boer, R;Cordwell, C;Marx, G;Fox, P;Hasovits, C;Rutovitz, J;Harold, M;Tran,

B;Wong, H-L;Gibbs, P

Title:

Demonstrating the feasibility of collecting secondary, de-identified data on Australian patients receiving treatment as part of a Medicine Access Programme

Date:

2020-01-01

Citation:

Lok, S. W., De Boer, R., Cordwell, C., Marx, G., Fox, P., Hasovits, C., Rutovitz, J., Harold, M., Tran, B., Wong, H. -L. \& Gibbs, P. (2020). Demonstrating the feasibility of collecting secondary, de-identified data on Australian patients receiving treatment as part of a Medicine Access Programme. INTERNAL MEDICINE JOURNAL, 50 (1), pp.99-104. https:// doi.org/10.1111/imj.14265.

Persistent Link:

http://hdl.handle.net/11343/275214 